\title{
Morbus haemolyticus neonatalis durch die irregulären Rhesus-Antikörper Anti-C und Anti-G in Kombination
}

\section{Hemolytic Disease of the Newborn due to Combined Rh Antibodies Anti-C and Anti-G}

Autor

Institute
K. Strathmann ${ }^{1}$, C. Dücker ${ }^{1}$, T. J. Legler ${ }^{2}$, T. Hoffmann ${ }^{1}$, R. E. Scharf

${ }^{1}$ Institut für Hämostaseologie, Hämotherapie und Transfusionsmedizin und Hemophilia Comprehensive Care Center, Universitätsklinikum Düsseldorf, Heinrich Heine Universität

${ }^{2}$ Abteilung für Transfusionsmedizin, Universitätsmedizin Göttingen, Georg-August-Universität

\section{Schlüsselwörter}

- Morbus haemolyticus neonatalis

- irreguläre Antikörper

- Anti-C

- Anti-G

- Rhesus-Prophylaxe

Key words

- hemolytic disease of the newborn

- irregular antibodies

- anti-C

- anti-G

- rhesus prophylaxis
Bibliografie

DOI http://dx.doi.org/ 10.1055/s-0032-1328116 Transfusionsmedizin 2013; 3 : 23-27 (c) Georg Thieme Verlag KG Stuttgart · New York · ISSN 2191-8805

\section{Korrespondenzadresse} Univ.-Prof. Dr. med. Rüdiger E. Scharf, F. A. H.A. Institut für Hämostaseologie, Hämotherapie und Transfusionsmedizin und Hemophilia Comprehensive Care Center, Geb. 12.49

Universitätsklinikum Düsseldorf Heinrich-Heine-Universität

Moorenstraße 5

40225 Düsseldorf

rscharf@uni-duesseldorf.de

\section{Zusammenfassung}

$\nabla$

Wir berichten über einen schweren Morbus haemolyticus neonatalis eines rhesusnegativen Kindes (Ccddee). Das Neugeborene wurde 2 Tage nach zeitgerechter vaginaler Entbindung der Mutter (G2, P2; Rhesusformel ccddee) notfallmäßig aus einem peripheren Haus in das Universitätsklinikum Düsseldorf verlegt. Bei Übernahme bot das Kind einen Icterus gravis (Ges.-Bilirubin $>30 \mathrm{mg} / \mathrm{dl}$ ) bei Hämolyse (LDH > $1500 \mathrm{U} / \mathrm{l}$ ), aber noch normwertigem Hämoglobin $(13,7 \mathrm{~g} / \mathrm{dl})$. Unter Austauschtransfusion mit dem 3-fachen kindlichen Blutvolumen fiel das Ges.-Bilirubin auf $18 \mathrm{mg} / \mathrm{dl}$, bei weitergeführter intensiver Phototherapie innerhalb von $24 \mathrm{~h}$ auf $12 \mathrm{mg} / \mathrm{dl}$. Nach erfolgreicher Behandlung einer bereits bei Aufnahme bestehenden Sepsis konnte das Kind in gutem Allgemeinzustand entlassen werden. Ursache des Morbus haemolyticus neonatalis war eine erst postpartal diagnostizierte Alloimmunisierung durch antierythrozytäre Antikörper der Spezifitäten Anti-C und Anti-G in Kombination. Zwar wurde bei der Mutter auswärts ein als Anti-D fehlinterpretierter Alloantikörper ab der 8.SSW mit steigendem Titer gefunden, aber keine erneute Rhesusprophylaxe durchgeführt. Diese Entscheidung stützte sich offenbar auf den molekulargenetischen Befund „keine fetalen D-spezifischen Nukleinsäurepolymorphismen“ und fehlende indirekte Hinweise einer fetalen Hämolyse im weiteren Schwangerschaftsverlauf. Unsere immunhämatologischen Untersuchungen ergaben, dass die Mutter vermutlich bereits in ihrer Erstschwangerschaft (Rhesusformel von P1 Ccddee) immunisiert worden war und nun einen weiteren irregulären Antikörper der Spezifität Anti-G entwickelt hatte. Die Besonderheit des G-Antigens liegt darin, dass es auf Erythrozyten exprimiert wird, welche das C- und/oder D-Antigen tragen. Durch diese Assoziation kann ein Anti-G-Antikörper wie eine Kombination aus Anti-C und Anti-D

\section{Abstract \\ $\nabla$}

We report on a severe hemolytic disease of a newborn typed Rh-negative (Ccddee). Two days after vaginal delivery of the mother (G2, P2; Rhesus formula ccddee) in the 39th week of gestation, the infant was admitted from a peripheral hospital to the University Medical Center Düsseldorf. At emergency admission, the newborn presented with icterus gravis (total serum bilirubin $>30 \mathrm{mg}$ / dl) and hemolysis (LDH > $1500 \mathrm{U} / \mathrm{l}$ ) but still normal hemoglobin $(13.7 \mathrm{~g} / \mathrm{dl})$. Upon exchange transfusion (3-fold of puerile blood volume), total serum bilirubin declined to $18 \mathrm{mg} / \mathrm{dl}$ and further decreased to $12 \mathrm{mg} / \mathrm{dl}$ within the next $24 \mathrm{hrs}$. upon concomitant intense phototherapy. After successful treatment of a sepsis diagnosed at admission, the infant could be discharged in good general condition. The hemolytic disease of this newborn was caused by alloimmunization due to combined anti-C and anti-G antibodies that had not been identified antepartum. Interestingly, at the 8th week of gestation, a maternal alloantibody with increasing titers had been detected by external screening and monitoring; however, this irregular antibody was misinterpreted as an "anti-D". No rhesus prophylaxis was administered again, based on a "Rh-negative" status of the fetus who had been genotyped prenatally. Moreover, no indirect signs of fetal hemolysis were detectable during pregnancy. Our immunohematological examinations after birth revealed that the mother had been presumably immunized during her first pregnancy (rhesus type of P1 Ccddee) and developed another irregular antibody of anti-G specificity. The $G$ epitope is expressed on red cells which carry $C$ and/or D antigen(s). Due to this association, an anti-G antibody can mimic the specificity anti-C plus anti-D and thus be erroneously assessed. Along with that, an anti-C plus anti-D reactivity pattern can result from various combinations of anti-C, anti-D and anti-G 
erscheinen und verkannt werden. Bei dem Reaktionsmuster „Anti-C und Anti-D“ sind unterschiedliche Kombinationen von AntiC, Anti-D und Anti-G in Betracht zu ziehen. Die genaue Differenzierung der Antikörperspezifitäten erfolgt durch Absorptionsund Elutionsuntersuchungen mit spezifischen Testerythrozyten. Bei Ausschluss eines Antikörpers der Spezifität Anti-D ist bei rhesusnegativen Schwangeren mit einem rhesuspositiven Feten unbedingt eine Rhesusprophylaxe indiziert. antibodies all of which have to be taken into consideration. A precise differentiation of antibody specificities can be performed by absorption and elution techniques using specific RBC. Detection of anti-C plus anti-D reactivity and concomitant exclusion of an anti-D antibody mandatorily requires rhesus prophylaxis (RhIg) in pregnant women being typed Rh-negative and carrying a Rhpositive fetus.

\section{Einleitung \\ $\nabla$}

Irreguläre antierythrozytäre Alloantikörper gegen Merkmale des Rhesussystems können einen Morbus haemolyticus neonatorum (MHN) auslösen. Da die fetalen Antigene des Rhesussystems bereits in der Frühphase der Gravidität exprimiert werden, reagiert eine rhesusnegative Schwangere - nach Einschwemmung kritischer Mengen Rhesus-positiven fetalen Bluts - auf diesen immunogenen Stimulus mit Bildung antierythrozytärer Antikörper, die zur Beladung und Elimination fetaler Erythrozyten führen. Wirksam sind Antikörper der Subklassen IgG1 und IgG3. Ursache eines MHN ist zumeist eine Alloimmunisierung der Mutter gegen das Rhesusmerkmal D des Fetus. Selten wird ein MHN durch Alloantikörper der Spezifitäten Anti-C, Anti-c, Anti-E und Anti-e hervorgerufen. Hierunter sind vor allem Anti-c-Antikörper klinisch relevant, die zu ähnlich schweren Verlaufsformen wie bei einem Anti-D-MHN führen können [1]. Eine Rarität stellt ein durch Anti-G verursachter MHN dar. Die Besonderheit des G-Epitops liegt darin, dass es nahezu regelhaft auf Erythrozyten exprimiert wird, welche das C- und/oder D-Antigen tragen [2]. Wir berichten hier über die immunhämatologische Konstellation, die einen schweren MHN auslöste, da der gefundene maternale anti-D-Antikörper bei einem rhesusnegativen Feten als irrelevant angesehen wurde, stellen die zielgerichtete Diagnostik vor und diskutieren Maßnahmen zur Prophylaxe und zur Therapie bei MHN.

\section{Kasuistik}

$\nabla$

Ein 2 Tage altes männliches Neugeborenes wurde unter dem klinischen Bild eines Icterus gravis in der Universitätskinderklinik Düsseldorf aufgenommen. Die 34-jährige Mutter (G2, P2) des Kindes ist deutscher Herkunft und trägt die Blutgruppe A Rhesus negativ (D neg., Rh-Formel ccddee). Bei ihrer vorausgegangenen Schwangerschaft wurde die übliche 2-zeitige Rhesusprophylaxe durchgeführt. Das 1. Kind der Patientin ist rhesusnegativ (D neg.) und hat ein isoliertes C-Merkmal (Rh-Formel Ccddee). Die nach den Mutterschaftsrichtlinien vorgeschriebene Antikörperdiagnostik wurde bei der erneuten Schwangerschaft zum Zeitpunkt der 8. SSW in einem externen Labor durchgeführt. Dabei wurde ein irregulärer antierythrozytärer Alloantikörper der Spezifität Anti-D (Titer $1: 128)$ im indirekten Coombs-Test (Gelkarte) gefunden. Der verantwortliche Gynäkologe veranlasste eine Pränataldiagnostik mit molekulargenetischer Untersuchung auf das fetale Rhesusmerkmal D aus mütterlichem Plasma. Dabei wurden, in Übereinstimmung mit dem postnatalen serologischen Ergebnis „Rhesus negativ (D neg.)“, keine D-spezifischen Nukleinsäurepolymorphismen nachgewiesen. Wie im Mutterpass festgehal- ten, wurde in Anbetracht des molekulargenetischen Untersuchungsergebnisses „Nachweis eines Rhesus-negativen (D neg.) Fetus" in der 2. Schwangerschaft auf eine Anti-D-Prophylaxe bei der Mutter verzichtet. Weiterhin findet sich dokumentiert, dass Verlaufskontrollen keinen Anhalt für eine Hämolyse beim Fetus erbrachten. In der 21.SSW wurde eine Doppler-Sonografie der fetalen A. cerebri media unter der Frage einer Flussbeschleunigung als Hinweis auf eine klinisch relevante Hämolyse durchgeführt. Die im Mutterpass dokumentierten Antikörpertiter (ohne Angabe von Spezifitäten) beliefen sich auf $1: 128$ (8. SSW), $1: 128$ (12.SSW), $1: 512$ (15.SSW) und 1: 512 (19. SSW). In der 39. SSW + 5 wurde die Zweitgravida vaginal von einem Jungen in einem Geburtshaus entbunden (Geburtsgewicht 3980 g, APGARIndex 9/10/10). Am 2. Tag postnatal wurde das Neugeborene „bei zunehmender Gelbfärbung“ einem niedergelassenen Kinderarzt vorgestellt, der es umgehend in die nächstgelegene Kinderklinik einwies. Von hier wurde das Kind bei einer Hyperbilirubinämie von $32,3 \mathrm{mg} / \mathrm{dl}$ notfallmäßig auf die Intensivstation des Zentrums für Kinder- und Jugendmedizin des Universitätsklinikums Düsseldorf verlegt. Befund bei Übernahme: eutropher Neugeborener in reduziertem Allgemeinzustand, ausgeprägt ikterisches Hautkolorit, sonst klinisch unauffälliger körperlicher Untersuchungsbefund. Laborergebnisse bei Aufnahme: Ges.-Bilirubin 30,55 (Norm < 7,0) mg/dl, direktes Bilirubin 3,14 $(<0,30) \mathrm{mg} / \mathrm{dl}$, indirektes Bilirubin 27,41 mg/dl, C-reaktives Protein 4,2 (>0,5) mg/ dl, Laktatdehydrogenase 1508 (<953) U/l, Leukozyten 23,5 (5,0-

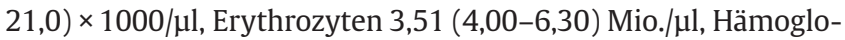
bin $13,7(13,0-22,0) \mathrm{g} / \mathrm{dl}$, Hämatokrit 40,8 (42,0-67,0) \%, MCV 116,2 (89,0-117,0) fl, MCH 39,0 (31,0-41,0) pg, MCHC 33,6 $(31,0-38,0)$ g Hb/dl, Thrombozyten $297(160-320) \times 1000 / \mu l$. Auf Grund des stark erhöhten Gesamt-Bilirubins wurde sofort eine intensive Phototherapie über $6 \mathrm{~h}$ eingeleitet. Allerdings konnte hierunter kein Rückgang der Hyperbilirubinämie erzielt werden, so dass eine Austauschtransfusion mit dem 3-fachen kindlichen Blutvolumen durchgeführt wurde. Die Konzentration des Gesamt-Bilirubins fiel auf $18,0 \mathrm{mg} / \mathrm{dl}$. Die fortgeführte Fototherapie konnte $24 \mathrm{~h}$ später bei Abfall des Gesamt-Bilirubins auf $12,2 \mathrm{mg} / \mathrm{dl}$ beendet werden. Ursache des bereits bei Aufnahme erhöhten und im Verlauf bis auf 4,8 mg/dl ansteigenden C-reaktiven Proteins war eine Sepsis (Nachweis Streptococcus agalactiae und Staphylococcus aureus in der Blutkultur), die nach Antibiogramm gezielt mit Ampicillin und Oxacillin behandelt wurde. Nach 2-wöchigem Klinikaufenthalt konnte das Kind in gutem Allgemeinzustand entlassen werden.

\section{Material und Methodik \\ $\nabla$}

Blutgruppenbestimmungen im AB0- und Rhesussystem und immunhämatologische Diagnostik (Antikörpersuchtest, Antikörperdifferenzierung, Antikörpertiterbestimmungen im indirekten 
Coombs-Test [indirekten Antiglobulintest, IAT]) und Durchführung des monospezifischen direkten Coombs-Tests (IgG und C3d) erfolgten mittels Gelkartentechnik (ID-Cards, ID-DiaCell, ID-DiaScreen, ID-DiaPanel, Bio-Rad, München). Zur Säureelution ge-

Tab. 1 Prinzip der Absorptions-Elutionsuntersuchung zum Nachweis von Anti-G, nach Issitt und Anstee [3].

\begin{tabular}{|c|c|c|}
\hline Antikörper im Patientenserum & $\begin{array}{l}\text { Anti-D } \\
\text { Anti-C } \\
\text { Anti-G }\end{array}$ & $\begin{array}{l}\text { Anti-D } \\
\text { Anti-C }\end{array}$ \\
\hline Testerythrozyten für 1 . Absorption & $C-D+G+$ & $C-D+G+$ \\
\hline Antikörper im 1. Eluat & $\begin{array}{l}\text { Anti-D } \\
\text { Anti-G }\end{array}$ & Anti-D \\
\hline Testerythrozyten für 2. Absorption & $C+D-G+$ & $C+D-G+$ \\
\hline Antikörper im 2. Eluat & Anti-G & kein Antikörper \\
\hline
\end{tabular}

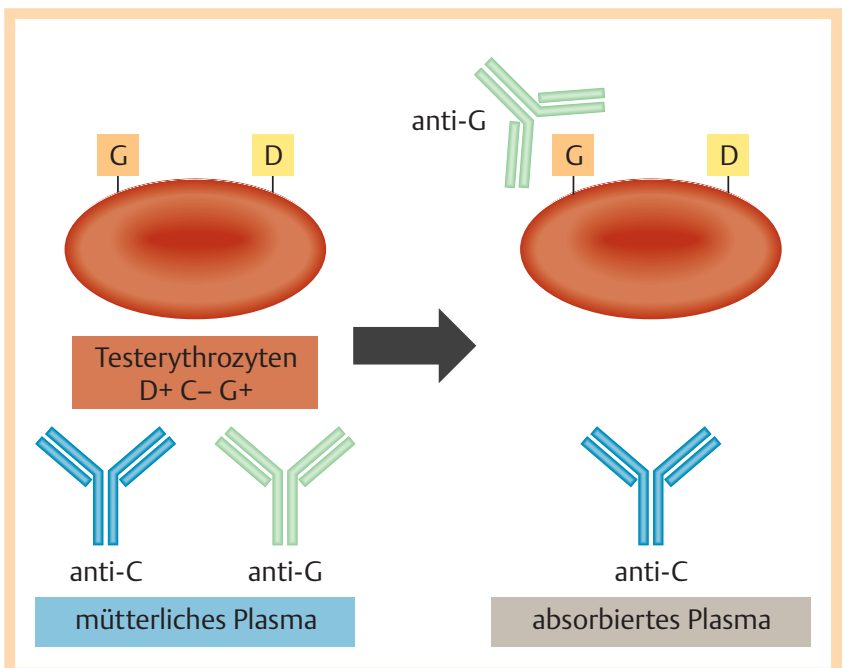

Abb. 1 Schritt 1: 1. Absorption des Patientenplasmas an ccD.ee-Testerythrozyten. Anti-G wird an den Erythrozyten absorbiert, Anti-C bleibt frei im Absorbat. bundener antierythrozytärer Antikörper aus EDTA-Blut des Neugeborenen setzten wir gebrauchsfertige Reagenzien ein (DiaCidel, Bio-Rad, München, vormals DiaMed, Köln). Die molekulargenetische Bestimmung des fetalen RHD-Merkmals aus mütterlichem Plasma wurde mittels Realtime PCR (ABI 7300, Applied Biosystems, Foster City, CA, USA) vorgenommen. Dazu wurde eine Duplex-PCR für Exon 5/Exon 7 zur Detektion von fetalem $R H D$ eingesetzt. Die Differenzialabsorptions- und Elutionsuntersuchungen führten wir mit mütterlichem Plasma und ccD.EEbzw. Ccddee-Testerythrozyten (Bio-Rad) durch. Hierzu erfolgte eine Bestätigungsdiagnostik im Immunhämatologischen Labor des DRK-Blutspendedienstes NSTOB, Institut Dessau. Zur Identifizierung des Anti-G-Antikörpers setzten wir die Methode nach Issitt und Anstee ein [3]. Hierzu wurden die im Patientenplasma vorhandenen Antikörper zunächst mit D-positiven und C-negativen Testerythrozyten absorbiert. Im nächsten Schritt erfolgte eine weitere Absorption des Eluats auf C-positive, D-negative Erythrozyten ( $\bullet$ Tab. 1). Der Untersuchungsablauf ist in $\boldsymbol{\otimes}$ Abb. 1 und 2 schematisch zusammengefasst.

\section{Ergebnisse und Diskussion}

\section{$\nabla$}

Beim Neugeborenen wurden die erythrozytären Blutgruppenmerkmale mit A Rhesus negativ (D neg.), Rhesusformel Ccddee, Kell (K) negativ bestimmt. Der polyspezifische direkte CoombsTest war 4-fach positiv; der monospezifische direkte CoombsTest zeigte eine starke IgG-Beladung (4+) der kindlichen Erythrozyten an. In der Elutionsuntersuchung der kindlichen Erythrozyten waren irreguläre Antikörper der vermeintlichen Spezifitäten Anti-C und Anti-D (IAT) nachweisbar. Im Antikörpersuchtest (IAT) mit kindlichem Plasma reagierten die Suchzellen I und II positiv. Im mütterlichen Plasma waren ebenfalls Antikörper der vermeintlichen Spezifitäten Anti-C und Anti-D (IAT) nachweisbar. Die jeweils im indirekten Coombs-Test mittels Gelkartentechnik bestimmten Anti-C- und Anti-D-Antikörper wiesen im mütterlichen Plasma eine Titerhöhe von 512 bzw. 256 auf.

cozyten $\mathrm{D}-\mathrm{C}+\mathrm{G}+$
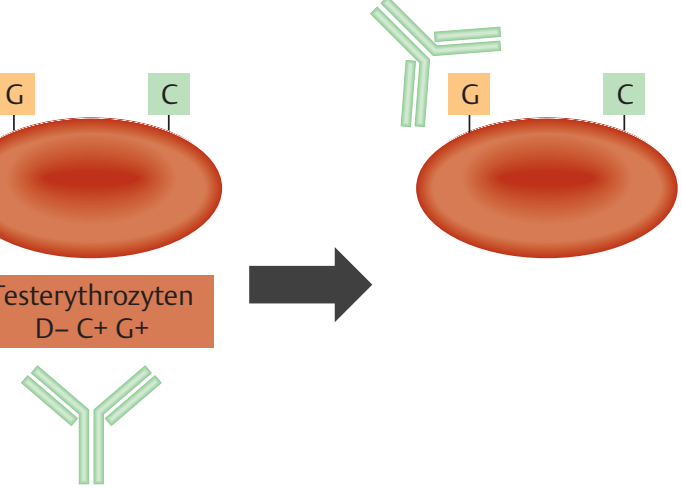

anti-G

Eluat

Absorbat

Abb. 2 Schritt 2: 2. Absorption. Hierbei wird Anti-G im Eluat aus der 1. Absorption an Ccddee-Testerythrozyten absorbiert. Es bleibt kein Antikörper frei im Absorbat.
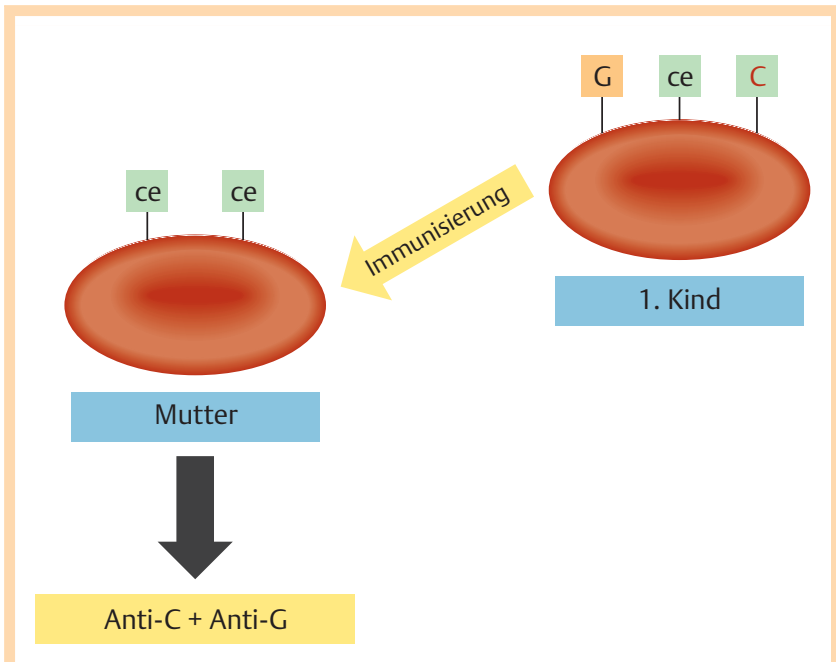

Abb.3 Immunisierungskonstellation bei der 1. Schwangerschaft der Patientin. Kind 1 hat die Rhesusformel Ccddee und ist G-positiv [6, 7]. Die Mutter (Rhesusformel ccddee) wird gegen die Rhesusantigene $C$ und $G$ immunisiert. 


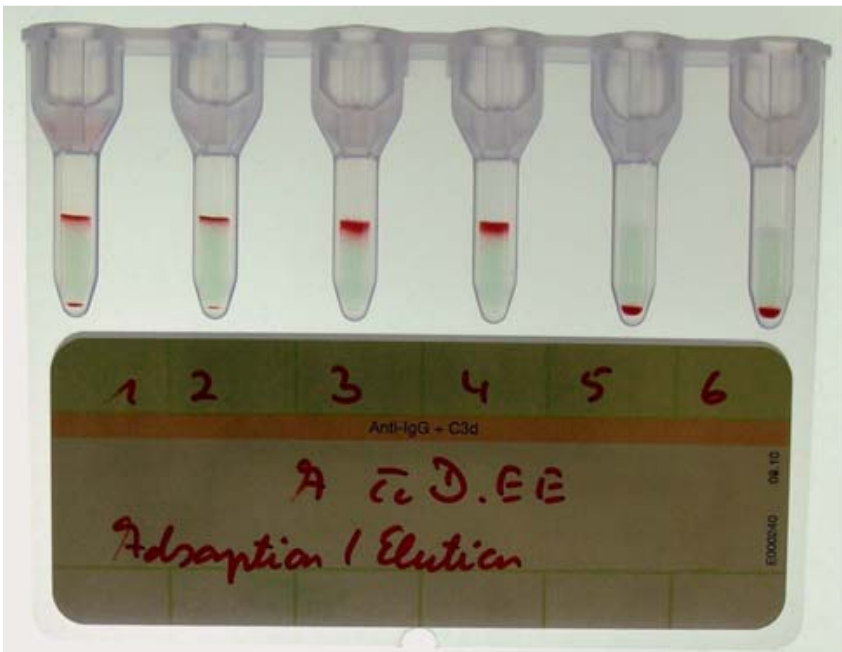

Abb. 4 Antikörperdifferenzierung nach Absorption und Elution mit ccD.EETesterythrozyten (indirekter Coombs-Test; Differenzierungspanel, Bio-Rad). Die Testzellen 1-3 und 8 sind D- und G-positiv, die Testzelle 4 ist C- und G-

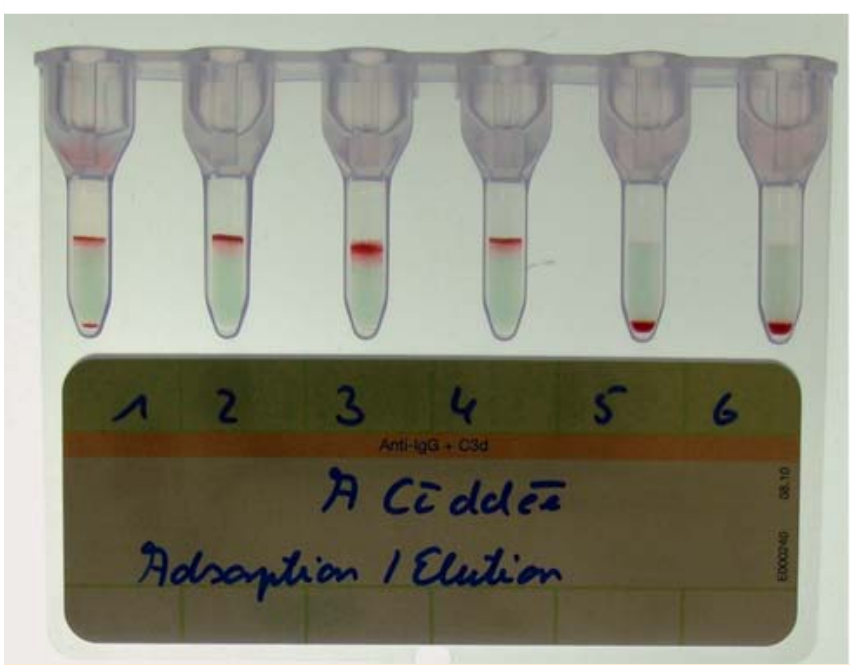

Abb.5 Antikörperdifferenzierung nach Absorption und Elution mit CcddeeTesterythrozyten (indirekter Coombs-Test, Differenzierungspanel, Bio-Rad). Die Testzellen 1-3 und 8 sind D- und G-positiv, die Testzelle 4 ist C- und G-

Während die Immunisierung der Mutter gegen das Merkmal C plausibel war ( $\bigcirc$ Abb. 3), erschien die Ätiologie einer Alloimmunisierung gegen das D-Antigen zunächst unklar: (1) Die Patientin hatte eine komplett negative Transfusionsanamnese, insbesondere keine zellulären Blutprodukte erhalten. (2) Eine denkbare Alloimmunisierung der Patientin gegen das Merkmal D während ihrer Erstschwangerschaft schied aufgrund der Rhesusformel des 1. Kindes (Ccddee) aus. Wir führten deshalb zusätzliche Differenzialabsorptions- und Elutionsuntersuchungen aus mütterlichem Plasma mit ccD.EE- und Ccddee-Testerythrozyten durch ( $\mathbf{A}$ Abb. 4 und 5). Aus der Tatsache, dass nach Absorption und Elution mit ccD.EE-Testerythrozyten ein Antikörper der Spezifität Anti-C und mit Ccddee-Erythrozyten ein Antikörper der Spezifität Anti-D detektierbar waren, ließ sich auf das Vorliegen eines Antikörpers der Spezifität Anti-G schließen. Die Diagnostik im Immunhämatologischen Labor des DRK-Blutspendediensts NSTOB, Institut Dessau, bestätigte die Antikörper Anti-C und Anti-G. Es fanden sich ein Anti-C- + Anti-G-Titer von 256 und

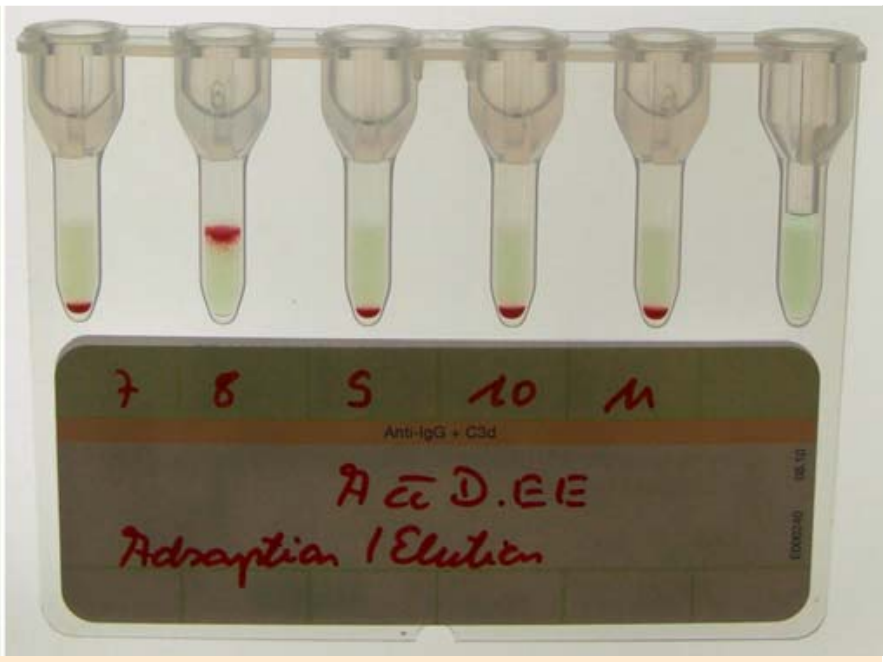

positiv, D-negativ. Nachweis einer Antikörperreagibilität gegen C- und D-positive Testzellen.

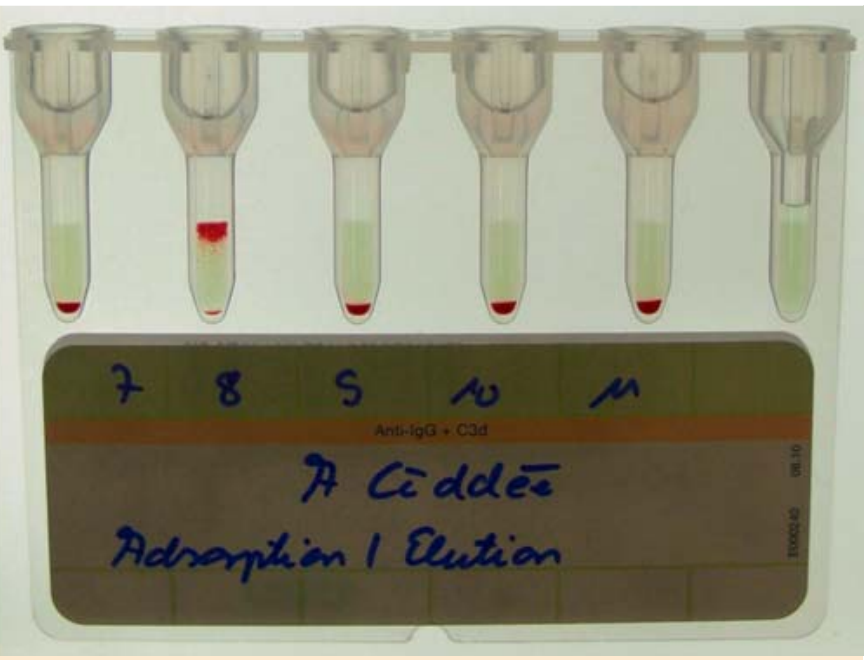

positiv, D-negativ. Aus dem Reaktionsmuster lässt sich auf die Antikörperspezifität Anti-G schließen.

ein Anti-G-Titer von 32. Gleichfalls bestätigt wurde, dass kein Anti-D-Antikörper vorlag.

Das Rhesusantigen G (ISBT-Symbol: RH12; ISBT-Nummer: 004012) [4] wurde erstmalig 1958 von Allen und Tippett beschrieben [5]. Wegweisend war der Befund, dass Anti-CD-Antiseren eine Agglutination von Erythrozyten eines C-negativen und D-negativen Blutspenders auslösten [5]. Molekulare Basis des GEpitops ist ein Thymin am Basenpaar 307 in Exon 2 des RHDoder $R H C E^{*} C$-Gens. Hieraus resultiert die Expression eines Serinrests an Position 103 des Rh-Proteins C oder D [6]. Dies bedeutet, dass Träger eines der folgenden Allele auch das G-Merkmal besitzen: $R H D, R H C E^{*} C^{*} e$ oder $R H C E^{*} C^{*} E[4,6,7]$. Für das Rhesusantigen $G$ sind folgende Häufigkeiten beschrieben: Kaukasier $84 \%$, Individuen afrikanischen Ursprungs 92\%, Asiaten 100\% [4]. Die klinische Relevanz als Ursache eines MHN dürfte variabel sein. So wurden bei Vorliegen eines Antikörpers der Spezifität Anti-G unauffällige bis schwere Verlaufsformen eines Morbus haemolyticus neonatorum berichtet $[8,9]$. 


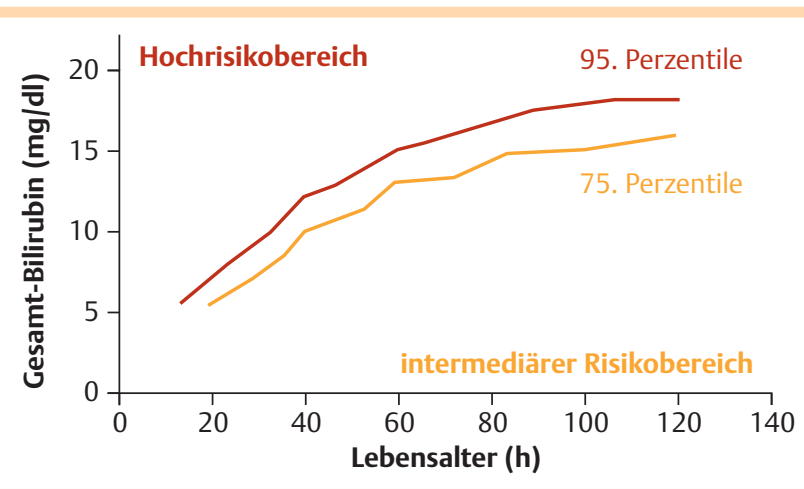

Abb. 6 Nomogramm mit altersbezogenen Konzentrationen des GesamtBilirubins bei reifen Neugeborenen zur Risikoabschätzung einer behandlungsbedürftigen Hyperbilirubinämie, nach Bhutani et al. [10], modifiziert.

Im vorliegenden Fall ergab sich bereits durch das Ausmaß der Hyperbilirubinämie die unmittelbar bestehende Indikation zur Therapie des Neugeborenen. Als Orientierung für die Behandlungsbedürftigkeit von Hyperbilirubinämien reifer Neugeborener dient ein Nomogramm ( $\bullet$ Abb. 6), das auch Gegenstand der AWMF-Leitlinie „Hyperbilirubinämie des Neugeborenen - Diagnostik und Therapie" ist $[10,11]$. In den ersten Tagen nach Geburt ist ein stetiger Anstieg unkonjugierten Bilirubins noch als physiologisch zu betrachten und zumeist ohne klinische Relevanz, solange der Serumspiegel die 95. bzw. 98. Perzentile mit Spitzenwerten der Bilirubinkonzentration von 18 bzw. $20 \mathrm{mg} / \mathrm{dl}$ nicht übersteigt [11]. Die beschriebene immunhämatologische Konstellation erklärt die Antikörper-induzierte Hämolyse des Kindes, das Ausmaß der resultierenden Hyperbilirubinämie von $>30 \mathrm{mg} / \mathrm{dl}$ $48 \mathrm{~h}$ nach Geburt und das unzureichende Ansprechen auf die intensive Phototherapie innerhalb von $6 \mathrm{~h}$.

\section{Fazit}

$\checkmark$

Der Nachweis eines irregulären Antikörpers der Spezifität Anti-C plus Anti-D macht bei Frauen im gebärfähigen Alter, insbesondere im Falle eingetretener Schwangerschaft, eine gezielte Spezialdiagnostik erforderlich. Bei scheinbar eindeutig gefundener kombinierter Spezifität gegen das RhD- und RhC-Antigen können - außer Anti-C + Anti-D - weitere Antikörperkonstellationen vorliegen: (1) Anti-C + Anti-D + Anti-G, (2) Anti-C + Anti-G, (3) Anti$D+$ Anti-G oder (4) Anti-G. Die exakte Differenzierung der Antikörperspezifitäten ist für die adäquate Rhesusprophylaxe relevant, allzumal bei fehlendem Nachweis bzw. Ausschluss des Alloantikörpers Anti-D. Je nach Befundkonstellation können sich wichtige Hinweise auf die klinische Bedeutung bzw. die zu erwartende Schwere eines Morbus haemolyticus neonatorum ergeben. So ist bei isoliertem Anti-G-Antikörper eher mit einem milden Verlauf zu rechnen, wie aus berichteten Einzelfällen ersichtlich wird $[8,9]$. Eine sorgfältige Erhebung der Transfusionsanamnese und Vorgeschichte vorausgegangener Schwangerschaften in Zusammenschau mit den Blutgruppenbefunden der Geschwisterkinder trägt zur zielgerichteten Diagnosefindung und korrekten Interpretation auch seltener Antikörperkonstellationen bei. In dem hier von uns berichteten Fall eines schweren Morbus haemolyticus neonatalis waren im Mutterpass nur Titerhöhen, aber keine Antikörperspezifitäten angegeben. Gerade die Untersuchung auf Antikörperspezifität(en) und deren Dokumentation sind zwingend geboten.
Bei retrospektiver Betrachtung der hier berichteten Kasuistik wird evident, dass der initial nachgewiesene Alloantikörper der scheinbaren Spezifität Anti-D in der auswärtigen Diagnostik zusammen mit dem molekulargenetischen Ergebnis eines RHD-negativen Fetus zur Fehleinschätzung eines „klinisch nicht relevanten Antikörperbefundes" führte. Es kann davon ausgegangen werden, dass bei umfassender Antikörperdifferenzierung im weiteren Verlauf der Schwangerschaft rechtzeitig die Spezifität „Anti-C plus Anti-D“ zu detektieren gewesen wäre. In Kenntnis dieses Befundes wäre bei zusätzlicher Bestimmung des fetalen RHCE*C-Merkmals aus mütterlichem Plasma die Gefahr eines Morbus haemolyticus fetalis bzw. neonatalis eher einzuschätzen gewesen mit der Konsequenz, die Entbindung in einer Klinik mit angeschlossenem Level-1-Perinatalzentrum zu veranlassen. Diese Vorkehrung zur bestmöglichen Versorgung eines Neugeborenen ist nach der aktuellen Leitlinie der zuständigen Fachgesellschaften bei Morbus haemolyticus fetalis bzw. neonatalis heute unerlässlich [12].

\section{Danksagung \\ $\nabla$}

Die Autoren danken Dr. Hartmut Kroll, DRK-Blutspendedienst NSTOB, Institut Dessau, für die serologische Bestätigungsdiagnostik.

\section{Interessenkonflikt \\ $\nabla$}

R.E. Scharf erhält Forschungsförderung von Baxter Deutschland und Bayer HealthCare und ist Gutachter und Berater von Pfizer und CSL Behring. Die anderen Autoren zeigen keine Interessenkonflikte an.

\section{Literatur}

1 Klein HG, Anstee DJ. Mollison's Blood Transfusion in Clinical Medicine. 11th ed. Oxford: Blackwell Publishing; 2005: 496-545

2 Goudemand $M$, Salmon $C$. Immunohématologie et Immunogénétique. Paris: Flammarion Médecine-Sciences; 1980

3 Issitt PD, Anstee DJ. Applied Blood Group Serology. 3rded. Miami: Montgomery Scientific Publications; 1998: 350-351

4 Reid ME, Lomas-Francis C, Olsson ML. The Blood Group Antigen FactsBook. San Diego: Academic Press; 2012: 218-219

5 Allen jr. FH, Tippett PA. A new Rh blood type which reveals the Rh antigen G. Vox Sang 1958; 3: 321-330

6 Faas BH, Beckers EA, Simsek S et al. Involvement of Ser103 of the Rh polypeptides in G epitope formation. Transfusion 1996; 36: 506-511

7 Lirochon J, Doinel C, Rouger P et al. Biochemical identification and characterization of the $\mathrm{G}$ antigen in the human blood group Rh. Immunology 1988; 64: 337-340

8 Cash K, Brown T, Strupp A et al. Anti-G in a pregnant patient. Transfusion 1999; 39: 531-533

9 Huber AR, Leonard GT, Driggers RW et al. Case report: moderate hemolytic disease of the newborn due to anti-G. Immunohematology 2006; 22: $166-170$

10 Bhutani VK, Johnson L, Sivieri EM. Predictive ability of a predischarge hour-specific serum bilirubin for subsequent significant hyperbilirubinemia in healthy-term and near-term newborns. Pediatrics 1999; 103: 6-14

11 Gesellschaft für Neonatologie und Pädiatrische Intensivmedizin. Leitlinie: Hyperbilirubinämie des Neugeborenen - Diagnostik und Therapie. Im Internet: www.awmf.org/leitlinien.html; Stand: 03/2010

12 Deutsche Gesellschaft für Gynäkologie und Geburtshilfe, Gesellschaft für Neonatologie und Pädiatrische Intensivmedizin, Deutsche Gesellschaft für Perinatale Medizin. Leitlinie: Indikation zur Einweisung von Schwangeren in Krankenhäuser der adäquaten Versorgungsstufe. Im Internet: www.awmf.org/leitlinien.html; Stand: 06/2008 